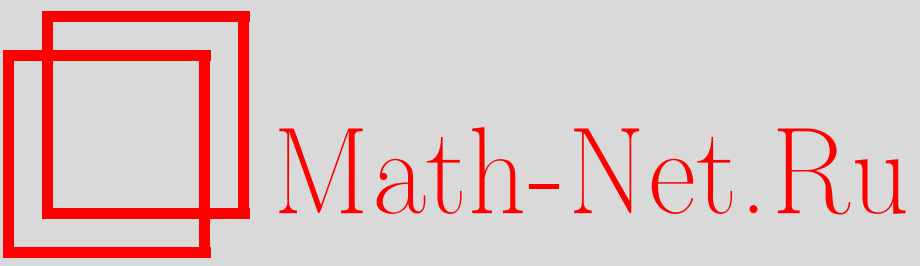

С. В. Нагаев, Новое доказательство абсолютной сходимости ряда Спицера, Теория вероятн. и ее примен., 2009, том 54, выпуск 1, 149-153

DOI: https://doi.org/10.4213/tvp2550

Использование Общероссийского математического портала Math-Net.Ru подразумевает, что вы прочитали и согласны с пользовательским соглашением

http://www.mathnet.ru/rus/agreement

Параметры загрузки:

IP : 3.95 .254 .165

26 апреля 2023 г., 10:58:57 


\title{
НОВОЕ ДОКАЗАТЕЛЬСТВО АБСОЛЮТНОЙ СХОДИМОСТИ РЯДА СПИЦЕРА ${ }^{1)}$
}

\begin{abstract}
Дается новое доказательство абсолютной сходимости ряда Спицера, основанное на неравенстве Берри-Эссеена. При этом оценивается сверху сумма ряда, составленного из абсолютных величин членов ряда Спицера.
\end{abstract}

Ключевье слова и фразы: ряд Спицера, оценка Берри-Эссеена, нормальный закон, абсолютная постоянная, постоянная Эйлера.

Пусть $X, X_{1}, X_{2}, \ldots, X_{n}, \ldots$ - независимые одинаково распределенные случайные величины с нулевым средним и конечной дисперсией $\sigma^{2}=\mathbf{E} X^{2}$. цера

Положим $S_{n}=\sum_{k=1}^{n} X_{k}, F(x)=\mathbf{P}(X<x)$. Б. Розен [1] доказал, что ряд Спи-

$$
\sum_{n=1}^{\infty} n^{-1}\left(\mathbf{P}\left(S_{n}>0\right)-\frac{1}{2}\right)
$$

абсолютно сходится.

Мы получим этот результат другим методом, используя единственно оценку Берри-Эссеена в центральной предельной теореме. Попутно мы оценим сверху сумму ряда

$$
\sum_{n=1}^{\infty} n^{-1}\left|\mathbf{P}\left(S_{n}<0\right)-\frac{1}{2}\right|
$$

Пусть $n_{1}=\min \left[n: \mathbf{E}\left\{X^{2} ;|X|<\sigma \sqrt{n}\right\}>\frac{3}{4} \sigma^{2}\right], n_{0}=\max \left[8, n_{1}\right]$.

Теорема. Справедливо неравенство

$$
\sum_{n=1}^{\infty} n^{-1}\left|\mathbf{P}\left(S_{n}<0\right)-\frac{1}{2}\right|<\frac{1}{2} \ln n_{0}+11 c_{0}+3.45
$$

где $c_{0}-$ абсолютная постоянная в оченке Берри-Эссеена.

Как показывает пример в конце настоящей заметки, от $\ln n_{0}$ в правой части неравенства (1) избавиться нельзя.

Если воспользоваться оценкой $c_{0}<0.7051$ из [2], то мы приходим к неравенству

$$
\sum_{n=1}^{\infty} n^{-1}\left|\mathbf{P}\left(S_{n}<0\right)-\frac{1}{2}\right|<\frac{1}{2} \ln n_{0}+12 .
$$

Д ок а з а т е л ь с т в о. Положим

$$
X_{j}(y)= \begin{cases}X_{j}, & \left|X_{j}\right| \leqslant y \\ 0, & \left|X_{j}\right|>y\end{cases}
$$

* Институт математики им. С. Л. Соболева, пр. Коптюга, 4, 630090 Новосибирск, Россия; e-mail: nagaev@math.nsc.ru,nagaevs@hotmail.com, nagaevs@academ.org

1) Работа выполнена при поддержке РФФИ (грант № 06-01-00069). 
Аналогично определяется $X(y)$. Пусть $S_{n}(y)=\sum_{k=1}^{n} X_{k}(y)$. Обозначим

$$
\begin{aligned}
& a(y)=\int_{|x|>y} x d F(x), \quad b^{2}(y)=\mathbf{E} X^{2}(y), \quad \sigma^{2}(y)=b^{2}(y)-a^{2}(y), \\
& B_{n}^{2}(y)=n \sigma^{2}(y), \quad \beta_{3}(y)=\mathbf{E}|X(y)|^{3}, \quad \gamma_{3}(y)=\mathbf{E}|X(y)+a(y)|^{3} .
\end{aligned}
$$

Согласно оценке Берри-Эссеена,

$$
\left|\mathbf{P}\left(S_{n}(y)<0\right)-\Phi\left(\frac{n a(y)}{B_{n}(y)}\right)\right|<c_{0} \frac{\gamma_{3}(y)}{\sigma^{3}(y)} n^{-1 / 2},
$$

где $\Phi$ - стандартный нормальный закон, $c_{0}$ - абсолютная постоянная.

Поскольку $\left|\mathbf{P}\left(S_{n}(y)<0\right)-\mathbf{P}\left(S_{n}<0\right)\right|<n \mathbf{P}(|X|>y)$ и

$$
\left|\Phi\left(\frac{n a(y)}{B_{n}(y)}\right)-\frac{1}{2}\right|<\frac{1}{\sqrt{2 \pi}} \frac{n|a(y)|}{B_{n}(y)},
$$

имеем

$$
\left|\mathbf{P}\left(S_{n}<0\right)-\frac{1}{2}\right|<c_{0} \frac{\gamma_{3}(y)}{\sigma^{3}(y)} n^{-1 / 2}+\frac{1}{\sqrt{2 \pi}} \frac{n|a(y)|}{B_{n}(y)}+n \mathbf{P}(|X|>y) .
$$

Заметим,что при $y>\sigma \sqrt{n}_{1}$ выполнено неравенство $|a(y)|<\sigma^{2} /(4 y)$ и, следовательно $\sigma^{2}(y)>\sigma^{2} / 2$. Не нарушая общности, мы можем считать, что $\sigma^{2}=1$.

Полагая в (2) $y=\sqrt{n}$ и производя суммирование, получаем оценку

$$
\begin{gathered}
\sum_{n=n_{0}+1}^{\infty} n^{-1}\left|\mathbf{P}\left(S_{n}<0\right)-\frac{1}{2}\right|<2 \sqrt{2} c_{0} \sum_{n=n_{0}+1}^{\infty} n^{-3 / 2} \gamma_{3}(\sqrt{n}) \\
+\frac{1}{\sqrt{\pi}} \sum_{n=n_{0}+1}^{\infty} \frac{a(\sqrt{n})}{\sqrt{n}}+\sum_{n=n_{0}+1}^{\infty} \mathbf{P}(|X|>\sqrt{n}) .
\end{gathered}
$$

Лемма. Если $\sigma^{2}=1$, то для любых $y \geqslant 2$

$$
\gamma_{3}(y) \leqslant \beta_{3}(y)+\frac{19}{8} .
$$

Д о к а з а т е л ь с т в о. Нетрудно видеть, что

$$
\gamma_{3}(y) \leqslant \beta_{3}(y)+3 \sigma^{2}(y)|a(y)|+3|a(y)|^{2} \mathbf{E}|X(y)|+|a(y)|^{3} .
$$

Далее $|a(y)|<\frac{1}{2}, \mathbf{E}|X(y)|<1$. Подставляя эти оценки в правую часть (5), мы получаем желаемый результат.

Продолжим доказательство теоремы.

Вследствие (4)

$$
\sum_{n=n_{0}+1}^{\infty} n^{-3 / 2} \gamma_{3}(\sqrt{n})<\sum_{n=n_{0}+1}^{\infty} n^{-3 / 2} \beta_{3}(\sqrt{n})+\frac{19}{8} \sum_{n=n_{0}+1}^{\infty} n^{-3 / 2} .
$$

Заметим, что при $k \geqslant n_{0}$

$$
\sum_{n=k+1}^{\infty} n^{-3 / 2}<2 k^{-1 / 2}
$$

Используя это неравенство, получаем

$$
\begin{aligned}
\sum_{n=n_{0}+1}^{\infty} n^{-3 / 2} \beta_{3}(\sqrt{n}) & =\sum_{k=n_{0}}^{\infty} \sum_{n=k+1}^{\infty} n^{-3 / 2} \int_{\sqrt{k}<|x| \leqslant \sqrt{k+1}}|x|^{3} d F(x)+\beta_{3}\left(\sqrt{n_{0}}\right) \sum_{n=n_{0}+1}^{\infty} n^{-3 / 2} \\
& <2\left(\sum_{k=n_{0}}^{\infty} k^{-1 / 2} \sqrt{k+1} \int_{\sqrt{k}<|x| \leqslant \sqrt{k+1}} x^{2} d F(x)+\int_{|x| \leqslant \sqrt{n_{0}}} x^{2} d F(x)\right) \\
& <3 / \sqrt{2}<2.13 .
\end{aligned}
$$


Из (6), (7) и последнего неравенства следует

$$
\sum_{n=n_{0}+1}^{\infty} n^{-3 / 2} \gamma_{3}(\sqrt{n})<3.81
$$

Оценим теперь $\sum_{n=n_{0}+1}^{\infty}|a(\sqrt{n})| / \sqrt{n}$. Очевидно, что $|a(y)| \leqslant v(y)$, где $v(y)=$ $\int_{|x|>y}|x| d F(x)$. Поскольку $n^{-1 / 2}<2(\sqrt{n}-\sqrt{n-1})$, имеем

$$
\begin{aligned}
\sum_{n=n_{0}+1}^{\infty} \frac{|a(\sqrt{n})|}{\sqrt{n}} & \leqslant \sum_{n=n_{0}+1}^{\infty} \frac{v(\sqrt{n})}{\sqrt{n}}<2 \sum_{n=n_{0}+1}^{\infty}(\sqrt{n}-\sqrt{n-1}) v(\sqrt{n}) \\
& <2 \int_{y>n_{0}} v(y) d y=2\left(\int_{|x|>n_{0}} x^{2} d F(x)-v\left(n_{0}\right) n_{0}\right)<2 .
\end{aligned}
$$

Наконец,

$$
\sum_{n=n_{0}+1}^{\infty} \mathbf{P}(|X|>\sqrt{n})<\sum_{n=1}^{\infty} \frac{v(\sqrt{n})}{\sqrt{n}}<2 .
$$

Комбинируя оценки (3) и (8)-(10), находим, что

$$
\sum_{n=n_{0}+1}^{\infty} n^{-1}\left|\mathbf{P}\left(S_{n}<0\right)-\frac{1}{2}\right|<11 c_{0}+2\left(1+\frac{1}{\sqrt{\pi}}\right)<11 c_{0}+3.13
$$

Нетрудно видеть, что

$$
\sum_{k=1}^{n_{0}} n^{-1}\left|\mathbf{P}\left(S_{n}<0\right)-\frac{1}{2}\right|<\frac{1}{2}\left(\ln n_{0}+\mathbf{C}+\frac{1}{2 n_{0}}\right),
$$

где $\mathbf{C}$ - постоянная Эйлера (см. [3, формула 0.131]).

Из (11) и (12) следует утверждение теоремы.

П р и м е р. Пусть $0<p<\frac{1}{2}$. Положим

$$
\mathbf{P}\left(X=p^{-1}\right)=p, \quad \mathbf{P}\left(X=(p-1)^{-1}\right)=1-p .
$$

Тогда $\mathbf{P}\left(S_{n}<0\right) \geqslant(1-p)^{n}$. Поскольку $\ln (1-p)>p /(p-1)$, то $(1-p)^{n}>e^{-2 n p}$. Следовательно, $\mathbf{P}\left(S_{n}<0\right)>e^{-2 n p}$. Отсюда

$$
\sum_{n=1}^{[1 /(4 p)]} n^{-1}\left|\mathbf{P}\left(S_{n}<0\right)-\frac{1}{2}\right|>\left(e^{-1 / 2}-\frac{1}{2}\right) \sum_{n=1}^{[1 /(4 p)]} n^{-1}>0.1 \ln \left[\frac{1}{4 p}\right] .
$$

Очевидно, что при $p<\frac{1}{8}$

$$
\ln \left[\frac{1}{4 p}\right]>\ln \frac{1}{4 p}+\ln (1-4 p)>\ln \frac{1}{4 p}-\ln 2=\ln \frac{1}{p}-3 \ln 2 .
$$

Если $p<1 / 8$, то $n_{0}<p^{-2}+1$. Отсюда $\ln n_{0}<2 \ln (1 / p)+1 / 64$, т.е.

$$
\ln \left[\frac{1}{4 p}\right]>\frac{1}{2} \ln n_{0}-3 \ln 2-\frac{1}{128} \text {. }
$$

Таким образом, при достаточно малых $p$

$$
\sum_{n=1}^{[1 /(4 p)]} n^{-1}\left|\mathbf{P}\left(S_{n}>0\right)-\frac{1}{2}\right|>0.03 \ln n_{0}
$$


3 а м е ч а н и е. На самом деле, как это нетрудно усмотреть из доказательства, справедливо более общее, нежели (1), неравенство

$$
\sum_{n=1}^{\infty} n^{-1}\left|\mathbf{P}\left(S_{n}<x \sigma \sqrt{n}\right)-\Phi(x)\right|<\frac{1}{2} \ln n_{0}+11 c_{0}+3.45 .
$$

Таким образом, функция распределения суммы независимых одинаково распределенных случайных величин с конечной дисперсией не может сходиться к нормальному закону сколь угодно медленно. 


\section{СПИСОК ЛИТЕРАТУРЫ}

1. Rosén $B$. On the asymptotic distribution of sums of independent identically distributed random variables. - Ark. Mat., 1962, v. 4, № 4, p. 323-332.

2. Шевцова И. Г. Уточнение верхней оценки абсолютной постоянной в неравенстве Берри-Эссеена. - Теория вероятн. и ее примен., 2006, т. 51, в. 3, с. 622-626.

3. Градштейн И. С., Рыжик И. М. Таблицы интегралов, сумм, рядов и произведений. М.: Физматгиз, 1963, 1108 с.

Поступила в редакцию

19.IX.2008 